\title{
Miscues on the "lack of MEF2A mutations" in coronary artery disease
}

W. by Weng et al. that claimed lack of MEF2A mutations in coronary artery disease (CAD) (1) and the accompanying commentary by Altshuler and Hirschhorn (2). They analyzed the exons and exon-intron boundaries of the $M E F 2 A$ gene in $300 \mathrm{CAD}$ patients and 300 elderly subjects by direct sequence analysis.

First, the new report actually found 1 patient with CAD who had a mutation (S360L) that is located in the critical transcriptional activation domain (3) and may affect the transactivation function of MEF2A. The mutation was not found in any control subject. Rather than following up its assessment experimentally, the investigators analyzed S360L quickly with computer software and claimed it to be benign, but computational analysis does not have any power to distinguish whether a variant is a disease-causing mutation or a polymorphism. The bias is quite apparent, and the title of the report, "Lack of MEF2A mutations in coronary artery disease," is inaccurate and inappropriate. When the data from the study by Weng et al. is taken together with data from our previous report of MEF2A mutations in sporadic cases of CAD (4), there are 5 of 507 individuals with CAD with $M E F 2 A$ mutations and 0 of 491 controls, which suggests a significant association between $M E F 2 A$ mutations and CAD $(P=0.03$, estimated by conservative Fisher exact test). The statistical analysis by Altshuler and Hirschhorn appears to be incorrect and the significance was inaccurately reported as $P>0.2(2)$.

Second, the phenotyping work in this new report for categorization of CAD is grossly inadequate. The investigators used resting echocardiograms in the 5 subjects they classified as "controls" with the 21-bp deletion. This test does not provide any insight regarding the presence of significant coronary atherosclerosis. Coronary artery disease is frequently asymptomatic, and its diagnosis relies on performance of a coronary angiogram. In all of our studies, we have used the angiographic evidence of disease as critical criteria for classification as a control (4-6). Furthermore, it is impossible to classify young individuals as unaffected controls, and this was done in the case of several individuals, including the 45-year-old controls of families 2 and 3 and the 37-year-old and 42-year-old individuals in family 1 (III:5 and III:6). Virtually all of the "controls" in the report by Weng et al. (1) should have been properly defined as "uncertain phenotype" with respect to the presence or absence of CAD. It is quite unreasonable for any conclusion regarding cosegregation of the 21-bp deletion of $M E F 2 A$ to be based on such poor phenotypic characterization.

Of note, the proband of family 1 had a transient ischemic attack (1), which raises the issue of atherosclerotic vascular disease, and only 2 other individuals in the family were old enough to be properly categorized - only 1 of these had an exercise stress test, and none had a coronary angiogram. Two individuals from the family without the 21-bp deletion are affected with CAD, but they may represent phenocopies, as CAD is very common (both parents of 1 affected individual, III: 1 , are normal, and the father of another affected individual, III:3, had a stroke and died at 46 years of age), and the disease can derive from the married-in spouses. In sum, careful analysis of family data suggests that the available clinical data are insufficient to make any conclusions and certainly does not support the author's conclusion that the 21-bp deletion does not cosegregate with the disease and does not cause CAD.

Third, the authors mistakenly suggest that our 21-bp deletion in MEF2A was associated with premature CAD and myocardial infarction (MI) (5). This was not at all the case (5). The age of onset for the 21-bp deletion in the Wang et al. report ranges from 35 to 68 years for CAD and 40 to 80 years for MI (5). Reduced penetrance is well known for many human diseases with autosomal dominant inheritance patterns including cardiovascular diseases such as Brugada syndrome and long QT syndrome. The phenotypic expression of a mutation varies in different families as well as in different members of the same family. For example, $45 \%$ of mutation carriers can be silent carriers, and incomplete penetrance in families can be as low as $12.5 \%$ for Brugada syndrome (7). The molecular mechanisms for reduced or incomplete penetrance are not clear. Modifier genes, environmental factors, gene-gene interactions, and gene-environment interactions may play a role. For a complex disease such as CAD with involvement of numerous environmental factors, genetic factors, and interactions among these factors, incomplete penetrance should not be unexpected and may explain why the 4 individuals over 60 years of age and with the 21 -bp deletion in the new report have not developed MI yet. The heterogeneity of European-American white individuals (who are known to be highly heterogeneous) may also be a contributing factor.

It is interesting that the 21-bp deletion is present in $0.15 \%$ of elderly subjects (1). The action of this deletion may be analogous to that of a variant of the cardiac sodium channel gene SCN5A, S1103Y, which is more prevalent in African Americans (13.2\%) than in other populations (8). S1103Y cosegregates with cardiac arrhythmia in some families and is associated with arrhythmia in the African American population; but many carriers do not develop arrhythmias $(8,9)$.

Identification of mutations of $M E F 2 A$ in CAD patients, but not in controls, is consistent with the hypothesis that MEF2A mutations are causative. In another 200 CAD patients, we have identified another potential mutation (T215A) (unpublished observations). Continued identification of MEF2A mutations in CAD population by other independent research groups and studies with knockout/knockin mice with $M E F 2 A$ mutations will provide further supportive evidence that $M E F 2 A$ is a disease-causing gene for $\mathrm{CAD}$ and reveal how $M E F 2 A$ defects lead to atherosclerosis.

In conclusion, identification of the 21-bp deletion of $M E F 2 A$, which cosegregates with CAD, in 1 large family (5), identification of multiple mutations in other CAD patients and families $(1,4)$, and functional data demonstrating the deleterious effect of mutations on the function of the MEF $2 \mathrm{~A}$ protein $(4,5)$ provide evidence that MEF2A is a disease-causing gene for CAD. The new report by Weng et al. (1) does not refute our findings. It advances the field by helping to 
establish the incidence of the 21-bp MEF2A deletion and pointing out the possibility of incomplete penetrance. But without proper phenotyping work and experimental biological assessment, it is a misleading report that unfortunately suggests a negative bias and premature dismissal of an important biologic underpinning of CAD.

\section{Qing Wang, Shaoqi Rao, and Eric J. Topol}

The Cleveland Clinic Foundation, Cleveland, Ohio, USA.

Conflict of interest: The authors have declared that no conflict of interest exists.
Address correspondence to: Qing Wang or Eric J. Topol, The Cleveland Clinic Foundation, Cleveland, Ohio, USA. E-mail: wangq2@ccf.org (Q. Wang) or topole@ccf. org (E.J. Topol).

J. Clin. Invest. 115:1399-1400. (2005). doi:10.1172/JCI25475.

1. Weng, L., et al. 2005. Lack of MEF2A mutations in coronary artery disease. J. Clin. Invest. 115:1016-1020. doi:10.1172/JCI200524186.

2. Altshuler, D., and Hirschhorn, J.N. 2005. MEF2A sequence variants and coronary artery disease: a change of heart? J. Clin. Invest. 115:831-833. doi:10.1172/JCI200524715

3. Yu, Y.T. 1996. Distinct domains of myocyte enhancer binding factor-2A determining nuclear localization and cell type-specific transcriptional activity. J. Biol. Chem. 271:24675-24683.
4. Bhagavatula, M.R., et al. 2004. Transcription factor MEF2A mutations in patients with coronary artery disease. Hum. Mol. Genet. 13:3181-3188.

5. Wang, L., Fan, C., Topol, S.E., Topol, E.J., and Wang, Q. 2003. Mutation of MEF2A in an inherited disorder with features of coronary artery disease. Science. 302:1578-1581.

6. Wang, Q., et al. 2004. Premature myocardial infarction novel susceptibility locus on chromosome 1P34-36 identified by genomewide linkage analysis. Am. J. Hum. Genet. 74:262-271.

7. Priori, S.G., et al. 2000. Clinical and genetic heterogeneity of right bundle branch block and ST- segment elevation syndrome: a prospective evaluation of 52 families. Circulation. 102:2509-2515.

8. Splawski, I., et al. 2002. Variant of SCN5A sodium channel implicated in risk of cardiac arrhythmia. Science. 297:1333-1336.

9. Chen, S., et al. 2002. SNP S1103Y in the cardiac sodium channel gene SCN5A is associated with cardiac arrhythmias and sudden death in a white family. J. Med. Genet. 39:913-915.

\section{Response to Wang et al.}

W e refer readers to the commentary by Altshuler and Hirschhorn (1) for an evaluation of current data on the relationship between sequence variation in $M E F 2 A$ and coronary artery disease (CAD). In addition, we wish to clarify several points.

First, as noted in our paper, we initially sequenced the coding regions and intronexon boundaries of MEF2A in 300 cases only. Missense changes identified in cases (potential "mutations") were then screened in 300 controls by selective sequencing. Therefore, including these data in a metaanalysis of $M E F 2 A$ sequence differences in cases and controls is inappropriate. Nevertheless, we should state that we observed 5 missense changes through our sequencing efforts ( 1 found only in CAD [S360L], 1 only in non$\mathrm{CAD}[\mathrm{P} 432 \mathrm{~L}]$, and 3 common to both groups [N263S, P432del, and I481V]). Interestingly, $\mathrm{N} 263 \mathrm{~S}$ is one of the reported causative "mutations" observed in 2 CAD cases (2), but it is also found in our control cohort (an 80-yearold male lacking clinical CAD), which argues against its role as a causative mutation.

Second, Wang et al. state that the definition of controls used in our study is inadequate to support valid inference regarding the effect of the 21-bp deletion in MEF2A on CAD. Clearly, we cannot exclude the possibility that the apparently healthy elderly individuals who served as controls in our study may have had occult CAD. However, inspection of the original pedigree (QW1576) in which the deletion was first identified (3) reveals that 9 of the 10 carriers of the deletion had clinically manifest disease before the age of 65 . In our study, none of the 3 individuals aged 65 or older carrying the 21-bp deletion had any symptoms of CAD. Therefore, the 3 independently ascertained elderly individuals in our study do not recapitulate the readily observable clinical phenotype originally ascribed to the deletion. It is worth mentioning that these 3 carriers have each recently undergone exercise stress tests and/or nuclear perfusion scans, all of which were completely normal (Duke treadmill score, low risk; normal rest and stress perfusion images), and therefore there is no justification for further invasive coronary angiography. Echocardiography ruled out right ventricular hypertrophy, an abnormality noted in murine Mef2a deficiency, and was not intended to determine the presence or absence of CAD.

Third, the concept of incomplete penetrance is only valid for mutations that are known to cause disease. The 21-bp deletion in MEF2A does not meet this criterion for the reasons noted by Altshuler and Hirschhorn. To date there is no evidence that the 21-bp deletion in MEF2A is any more strongly associated with CAD than are other completely linked sequence variants within the originally reported interval (3). Therefore, unless additional evidence firmly connects $M E F 2 A$ sequence variation and $C A D$ risk, the identification of apparently healthy 21-bp deletion carriers cannot be ascribed to incomplete penetrance.

Finally, we have no vested interest in $M E F 2 A$ : we do not have grant support or any relationships, fiduciary or otherwise, that are in any way contingent on the outcome of the study. In terms of follow-up studies, a positive result would clearly have been far more interesting and of greater benefit to our laboratories (and was the original goal of this study). Unfortunately this was not the case, and the existing data currently do not support a significant role for MEF2A mutations as a cause of CAD.

\section{Li Weng, ${ }^{1}$ Jonathan Cohen, ${ }^{2}$ Ruth McPherson, ${ }^{3}$ and Len A. Pennacchio'}

${ }^{1}$ US Department of Energy Joint Genome Institute, Walnut Creek, California, USA. ${ }^{2}$ University of Texas Southwestern Medical Center, Dallas, Texas, USA. ${ }^{3}$ University of Ottawa Heart Institute, Ottawa, Ontario, Canada.

Conflict of interest: The authors have declared that no conflict of interest exists.

Address correspondence to: Len A. Pennacchio, US Department of Energy Joint Genome Institute, Walnut Creek, California, USA. E-mail: LAPennacchio@lbl.gov.

J. Clin. Invest. 115:1400 (2005). doi:10.1172/ JCI25476.

1. Altshuler, D., and Hirschhorn, J.N. 2005. MEF2A sequence variants and coronary artery disease: a change of heart? J. Clin. Invest. 115:831-833. doi:10.1172/JCI200524715.

2. Bhagavatula, M.R., et al. 2004. Transcription factor MEF2A mutations in patients with coronary artery disease. Hum. Mol. Genet. 13:3181-3188.

3. Wang, L., Fan, C., Topol, S.E., Topol, E.J., and Wang, Q. 2003. Mutation of MEF2A in an inherited disorder with features of coronary artery disease. Science. 302:1578-1581. 